\title{
Evaluación de la Huella Hídrica del Lirio Japonés (Hemerocallis)
}

\section{The Water Footprint of Japanese Lily (Hemerocallis)}

\section{Yuri S. Vanegas Rojas ${ }^{1}$, Lorena M. Vera Ramírez², Jesús A. Torres Ortega ${ }^{3}$}

\author{
Ingeniería Ambiental y Sanitaria, Universidad de La Salle, Bogotá, Colombia \\ 'yvanegas85@unisalle.edu.co, \\ ${ }^{2}$ Ivera14@unisalle.edu.co \\ ${ }^{3}$ jatorres@unisalle.edu.co
}

Recibido: 7/07/2013 • Aprobado: 02/09/2013

\section{Resumen}

Colombia es uno de los países que se caracteriza por su gran riqueza en biodiversidad y fuentes hídricas; así mismo es uno de los 10 países con mayor disponibilidad de este recurso en el mundo. Los seres humanos consumen agua diariamente en todo lo que realizan (bienes, productos, servicios). Es así, como la huella hídrica se utiliza como un indicador del consumo directo o indirecto del agua, tanto en el ámbito doméstico como en el industrial. Esta permite determinar el volumen total de agua dulce que se emplea para la elaboración de bienes, en servicios consumidos por los individuos o en una actividad en particular. El objetivo de esta investigación se centró en evaluar la huella hídrica para la producción de flores de Lirio japonés (Hemerocallis) en un predio del municipio de Rondón - Boyacá (Colombia). La propuesta metodológica planteada por los autores para este estudio consta de tres fases: el diagnóstico, el modelamiento y el análisis de resultados. El resultado obtenido para la huella hídrica azul es $1.76 \times 10-4 \mathrm{~L} /$ tallo, para la huella verde es $1 \times 10-8 \mathrm{l} /$ tallo y para la huella gris es $2.06 \times 10-4 \mathrm{~L} /$ tallo. Finalmente, una huella hídrica total es de 3,82×10-4L/tallo. En conclusión, la huella hídrica total del cultivo de Lirio japonés (7.64×10-3m3/ton) en comparación con la huella hídrica por producción de flores (900m3/ton), aporta tan solo el $0.00085 \%$ del total, por lo cual se puede afirmar que este cultivo es uno de los que tiene un menor aporte en dicha huella total para la producción de flores en Colombia.

Palabras clave: agua, azul, biodiversidad, cultivo, huella, hídrica, gris, Iluvia, verde, virtual. 


\section{Abstract}

Colombia is a country that is rich in biodiversity and water sources, one of the 10 countries with greater availability of water in the world. Humans spend water every day in everything we consume (belongings, products and services), and it is required for their production. The water footprint are used as an indicator of water used both domestic by consumers and industry, directly and indirectly, in which the total volume of fresh water used for the production of products and services consumed by individuals or a particular activity services determined. The objective is to evaluate the water footprint of Japanese lily flowers production (Hemerocallis) in an area of the municipality of Rondón (Boyacá - Colombia). The proposed methodology consists of three phases, initially is the diagnostic, follow of modeling and finally the analysis of results obtained in this study. The results obtained for the blue water footprint is $1.76 \times 10-4 \mathrm{l} / \mathrm{stem}$ for green footprint is $1 \times 10-8 \mathrm{~L} / \mathrm{stem}$ gray footprint is $2.06 \times 10-4 \mathrm{~L} /$ stem and finally a total water footprint 3,82×10-4 L/stem. In conclusion total water footprint Japanese lily cultivation (7.64×10-3 m3/ton) compared to the water footprint for flower production (900m3/ton), contributes only $0.00085 \%$ of the total, which said that this culture is one that has a lower contribution to this total footprint for flower production in Colombia.

Keywords: biodiversity, blue, grey, green, growing, rain, virtual, water

\section{INTRODUCCIÓN}

El área de estudio es el municipio de Rondón, uno de los 123 municipios del departamento de Boyacá, Colombia. Se encuentra ubicado en la provincia de Márquez a $60 \mathrm{Km}$ de Tunja y a 130 Km de Bogotá. Limita al norte con Toca, Siachoque, Viracachá y Ciénega; al Oriente con Pesca; al Sur con Zetaquirá y al Occidente con Ramiriquí [1]. El municipio de Rondón cuenta con una cuenca central correspondiente al río Mueche, que es alimentada por tres subcuencas y 22 microcuencas, las cuales surten al municipio. La quebrada La Puerquera es la fuente principal de abastecimiento hídrico de la zona de estudio.

El objetivo principal de este proyecto se centró en la evaluación de la huella hídrica para la producción de flores de Lirio japonés (Hemerocallis) en un predio del municipio de Rondón. Para tal fin, se realizó el diagnóstico de las etapas del proceso productivo de dicho cultivo y se establecieron los valores de las huellas hídricas azul, verde y gris del mismo. De la misma forma, se calculó la huella hídrica total en la producción de Lirio japonés.
Los resultados obtenidos muestran una visión general del valor de las huellas hídricas (azul, verde, gris y total) para el cultivo de Lirio japonés y sus aportes a la huella hídrica por producción de flores en Colombia.

Se encontró que la huella hídrica total del Lirio japonés no excede el $10 \%$ de la huella hídrica de producción de flores en Colombia. Con esto se puede plantear la siguiente pregunta: ¿es posible determinar la cantidad de agua necesaria para la producción de Lirio japonés (Hemerocallis) por medio del cálculo de la huella hídrica total?

Por lo anterior, nace la necesidad de visualizar y destacar la cantidad de agua dulce que pequeños empresarios colombianos utilizan para la producción de Lirio japonés en el municipio de Rondón (vereda el Ricaurte), con el fin de cuantificar los efectos de su consumo y comercio, sobre el uso del recurso hídrico y el suelo.

La huella hídrica en el sector floricultor presenta una información clara a lo largo del tiempo 
sobre la utilización de este recurso para diversos fines humanos y, por ende, genera la base para la evaluación local de su impacto ambiental, social y económico, buscando así estrategias o alternativas que permitan reducir el volumen de agua en los diferentes cultivos. Para tal fin, se utiliza una metodología cuantitativa, obteniendo así las variables correspondientes para cada una de las aguas virtuales: verde, azul y gris, contando, además, con los datos suministrados por la administración del cultivo.

Las técnicas y estrategias de recolección que se tuvieron en cuenta para la realización del proyecto son: listas de chequeo para la recolección de la información necesaria de cada una de las fases del proceso productivo del Lirio japonés; recolección de información secundaria sobre la climatología de la zona de estudio por medio del Ideam (Instituto de Hidrología, Meteorología y Estudios Ambientales de Colombia); toma de muestras representativas y análisis en el laboratorio. Así mismo, se tuvieron en cuenta el procedimiento para la determinación de la DQO (método Hach) del protocolo académico química ambiental, y estudios de calidad de la Universidad de la Salle y la normatividad pertinente [2]. Para el modelamiento, se contó con el software Cropwat 8.0 propuesto por la FAO en el año 2010. Este programa permitió determinar la evapotranspiración del cultivo de Lirio japonés y los requerimientos necesarios de agua y riego con base en los datos climatológicos obtenidos anteriormente. Para la correcta utilización del programa se manejaron los diferentes documentos y manuales que este programa contiene.

\section{Marco Teórico}

Colombia es uno de los países que posee una gran riqueza en biodiversidad y fuentes hídricas, y uno de los diez con mayor disponibilidad de recurso hídrico en el mundo [3]; sin embargo, se explota en grandes cantidades para la elaboración y producción de bienes y servicios, extracción de minerales y producción agropecuaria, entre muchas otras actividades que se desarrollan para satisfacer las necesidades de la sociedad. Los seres humanos consumen agua diariamente para la ducha, preparar alimentos, lavar utensilios, la ropa, o simplemente para beberla directamente; pero también se gasta gran cantidad en todo lo que se produce (bienes, productos, servicios). A escala global, aunque el agua se utiliza, en su gran mayoría, en la producción agrícola, también se consumen volúmenes sustanciales en los sectores industriales y domésticos [4]. Chapagain \& Hoekstra [5] han mostrado el empleo de agua en productos que pueden ayudar a la comprensión del carácter global que esta tiene en cuanto a la cuantificación de los efectos de consumo y el su comercio [5], [6], [7].

La huella hídrica $(\mathrm{HH})$ es un indicador del empleo del recurso hídrico que mira, no solo el empleo directo del consumidor o el productor, sino también su empleo indirecto [8].

Otro concepto importante relacionado con la huella hídrica un indicador multidimensional; depende del lugar y del tiempo, y permite identificar las relaciones socio ambientales respecto del agua; está orientada especialmente hacia las actividades socioeconómicas. Este concepto ayuda a visualizar el uso oculto del agua de diferentes productos y a comprender los efectos del consumo y el comercio frente al agua y su disponibilidad [3].

El concepto se basa en la teoría de agua virtual (AV) que fue definida a principios de la década de los noventa como el agua contenida en un producto, entendiendo por tal, no únicamente la cantidad física contenida en el producto, sino la cantidad de agua que ha sido necesaria para generar dicho producto. Así definida, el AV se configuraba como indicador físico en términos de agua de la producción de un bien o servicio [9]. El agua virtual fue desarrollada al estudiar la posibilidad de importar agua virtual (en lugar de agua real) como una solución parcial a los problemas de escasez de agua en el Oriente Medio. Si un país exporta un producto con un alto consumo de agua a otro país, exporta agua en forma virtual. 
De este modo, algunos países podrían apoyar a otros países en sus necesidades de agua. Para los países con escasez de agua podría ser atractivo disminuir presiones a su recurso hídrico mediante la importación de productos altamente demandantes de agua, en lugar de producirlos [9]. La huella hídrica de un sistema, de un individuo o de una comunidad se divide en tres componentes: azul, verde y gris (véase Fig. 1). La huella verde representa la precipitación sobre el cultivo que no provoque escorrentía o se sume a las aguas subterráneas ya que parte de ella se evaporará o se fijará a las plantas. La huella azul corresponde al volumen de agua captada de fuentes superficiales o subterráneas para cubrir la demanda de agua no satisfecha a causa de un déficit en la disponibilidad de agua procedente de la lluvia; además, es la cantidad de agua disponible en determinado periodo que se consume y que no es devuelta a la misma cuenca hidrográfica [3]. Y la huella gris se define como el volumen de agua requerida para asimilar residuos o cargas de contaminantes (herbicidas, abonos y demás) a través de la dilución de los mismos, hasta que la concentración del agua alcance la norma establecida para su calidad. Actualmente, las huellas hídricas verde y azul se estiman utilizando el modelo de simulación agronómico Cropwat.

\section{Materiales y Métodos}

De conformidad con la metodología estándar planteada por Water Footprint Network (WFN), se llevaron a cabo tres fases para la obtención de información.

La primera fase consistió en realizar un diagnóstico y la identificación de diferentes factores que actúan en el proceso productivo del Lirio japonés, tales como el volumen de agua necesaria para la producción, temperatura y humedad para la estabilidad del cultivo, cantidad de agroquími$\cos$ y plaguicidas manejados en el proceso productivo, volumen de vertimientos y energía necesaria para la producción.

Como material de apoyo, para la realización de esta etapa, se utilizaron listas de chequeo para la recolección de la información necesaria de cada una de las fases del proceso productivo. Fue indispensable contar con la recolección de información secundaria sobre la climatología de la zona de estudio para los cálculos de las huellas hídricas.

Para la determinación de la huella hídrica verde se realizó una toma de muestra del suelo si-

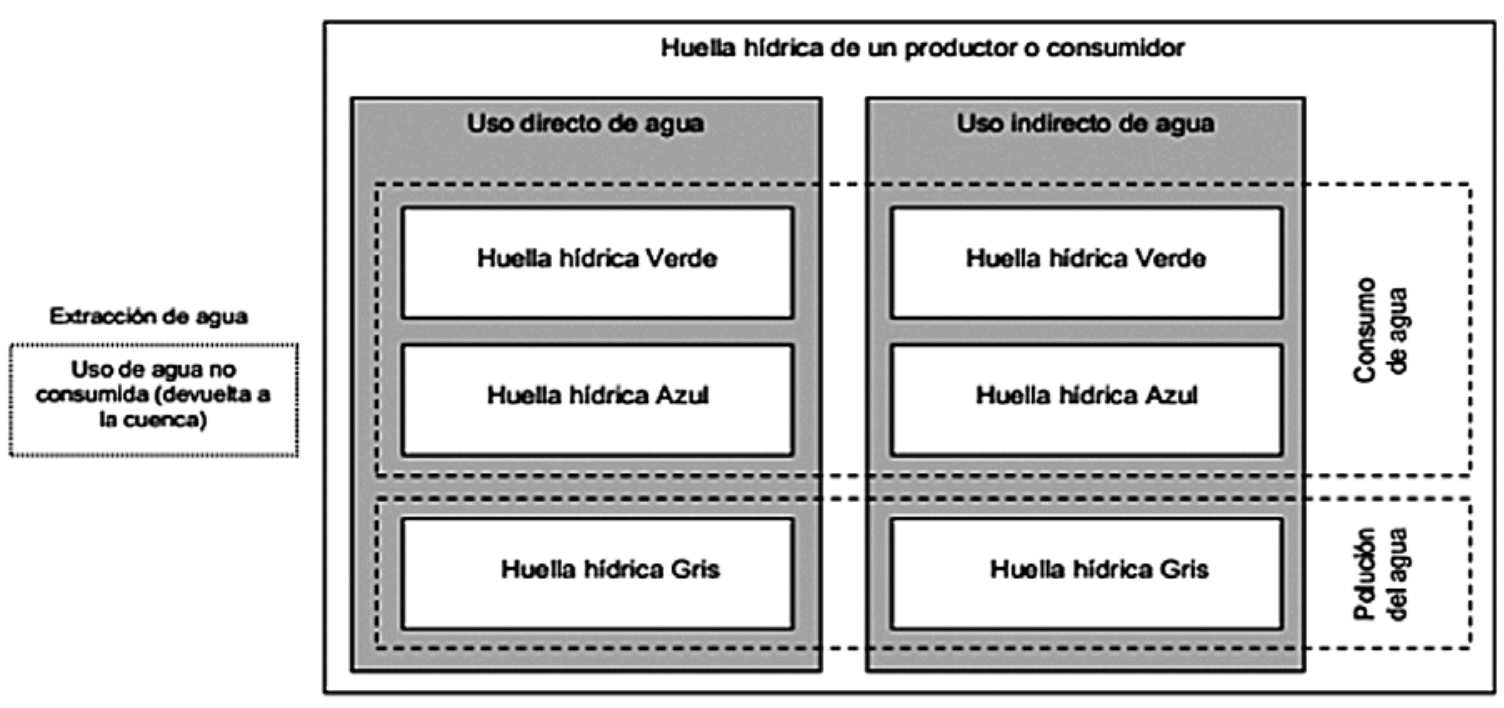

Fig. 1 Representación esquemática de los componentes de la huella hídrica [7]. 
guiendo la metodología planteada por WFN, teniéndose en cuenta el tiempo total de la prueba que es de 90 minutos (1 hora y media) [10]: se realizó la limpieza del área que se estipuló como punto de muestreo, removiendo las plantas y hojarasca que allí se encontraba. Se introdujo parcialmente un anillo de 6 pulgadas (6") de diámetro en la superficie de suelo, con una altura promedio de $50 \mathrm{~cm}$, tal y como se muestra en la Fig. 2: vaciar un volumen determinado de agua destilada, para que esta se infiltre en el suelo; a medida que el agua se va infiltrando, se lleva un registro del descenso de la columna de agua, tomando el tiempo y la altura de la misma.

Esta lectura del descenso del nivel de agua se realizó cada 5 minutos durante los 20 minutos. En el caso que el agua sea absorbida antes de terminarse la prueba, se debe añadir más agua (este proceso de llenado se repite tantas veces como sea necesario hasta concluir con la prueba de infiltración) o esperar hasta que la columna de agua se estabilice dentro del anillo.

Con los datos obtenidos se calculó la diferencia del nivel en cada uno de los intervalos de tiempo. Posteriormente, se determinó la cantidad de agua retenida por el suelo y la velocidad con la que esta se infiltró.

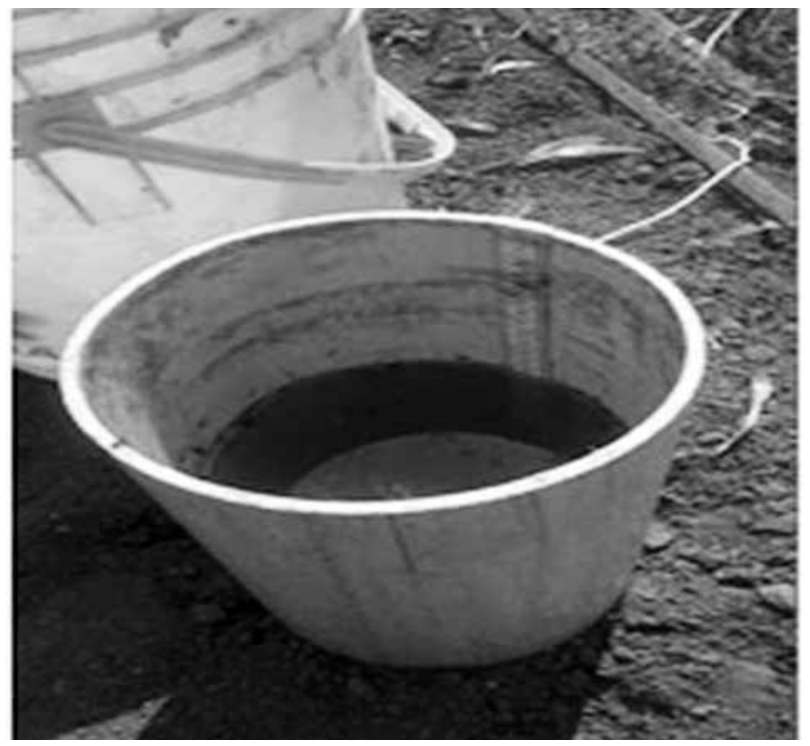

Fig. 2 Prueba de infiltración.
La ecuación con la cual se define el comportamiento de la infiltración es:

$$
Q=315.5 *\left(\frac{h}{t}\right)^{(1 / 2)}
$$

La Ecuación 1 representa el comportamiento de infiltración, en donde:

$\mathrm{Q}=$ Tasa de infiltración en $\mathrm{L} / \mathrm{m} 2$ día.

$\mathrm{H}=$ Descenso del nivel de agua en el tiempo de la prueba $(\mathrm{mm})$.

$\mathrm{t}=$ Tiempo demandado para el descenso de nivel de agua expresado en segundos.

Para el análisis de las muestras en el laboratorio se tuvo en cuenta el procedimiento para la determinación de la DQO (método Hach) del protocolo académico química ambiental y estudios de calidad de la Universidad de la Salle.

El método de Hach es un procedimiento muy sencillo que consiste en poner $2 \mathrm{ml}$ de muestras en las celdas Hach; posteriormente, en el digestor por 2 horas a $148^{\circ} \mathrm{C}$ y luego dejar enfriar en la gradilla y leer en el equipo.

La segunda fase de la investigación consistió en el modelamiento de cada una de las huellas hídricas (verde, azul y gris). Con esto se determinó la huella hídrica total del cultivo de Lirio japonés. Para este fin se utilizó el software Cropwat 8.0 propuesto por la $\mathrm{FAO}$ en el año 2010 , ya que este programa permite determinar la evapotranspiración del cultivo de Lirio japonés, requerimientos necesarios de agua y riego con base en los datos climatológicos obtenidos anteriormente.

Para la correcta utilización del programa en mención se manejaron los manuales Evapotranspiración de Cultivos: Guías para la determinación de los requerimientos de agua de los cultivos [11] y Cropwat 8.0 [12]; en estos manuales se encuentran consignadas cada una de las fórmulas que se emplearon para el modelamiento de los parámetros e información relevante, las cuales se resumen en la Tabla I. 
TABLA I

Resumen Información de Cálculos de las Huellas Hídricas

\section{Característica}

Huella hídrica verde

WF green [m3/ton]

Huella hídrica azul

WF blue, [m3/ton]

Huella hídrica gris

WF gray [m3/ton]

\section{Fórmula}

(2)

$\mathrm{Y}=$ Rendimiento del cultivo, expresado en tallos/ha.

$\mathrm{CWU}=$ Uso de Agua del Cultivo, expresado en m3/ha.

(3)

$Y=$ Rendimiento del cultivo, expresado en tallos/ha.

$\mathrm{CWU}=$ Uso de Agua del Cultivo, expresado en m3/ha.

(4)

Cantidad aplicada de productos químicos para el campo por hectárea (AR, $\mathrm{kg} / \mathrm{ha}$ ) Fracción $(\alpha)$ de lixiviación y escorrentía.

Concentración máxima aceptable ( $\mathrm{C} \max , \mathrm{kg} / \mathrm{m} 3)$

Concentración natural para el contaminante considerado (C nat, kg/m3)

Rendimiento del cultivo (Y, ton/ha).

Huella hídrica total

(5)

Fuente: los autores

Huellas hídricas verde y azul: se refiere al uso de agua de los cultivos (CWU, m3/ha), la cual se calculó teniendo en cuenta la acumulación de la evapotranspiración diaria (ET, mm/día) durante el período de crecimiento completo [12]. Para la determinación de la huella hídrica azul se aplicó la fórmula expuesta anteriormente que utiliza la resta de los requerimientos hídricos del cultivo con los valores de precipitación efectiva (Ecuación 2).

En cuanto a la obtención de información de datos climatológicos de la zona, los cuales fueron necesarios para el cálculo del componente de la huella hídrica verde, fue indispensable contar con los siguientes parámetros: humedad relativa, temperatura media mensual, precipitación media mensual, velocidad media del viento y la fracción de insolación de la zona. Estos datos fueron suministrados por la estación meteorológica Rondón, la más cercana a la zona de estudio.

Los datos sobre las características del cultivo de Lirio japonés, tales como, la fecha de siembra, la duración de etapas de crecimiento, el coeficiente del cultivo (Kc), la profundidad radicular, la altura máxima del cultivo y la fracción de agota- miento hídrico $(p)$, suministrados por la empresa en la cual se encontraba el cultivo (Ecuación 3), fueron definitivos para la determinación de la huella hídrica azul.

En cuanto al coeficiente del cultivo (Kc), este valor depende de las características anatómicas, morfológicas y fisiológicas de la planta; debido a esto varía según el estado vegetativo de la planta y del clima de la zona; también depende de la capacidad de extracción de agua del suelo que posee la planta a medida que esta se va desarrollando desde su siembra hasta su cosecha. Estos se identifican en tres valores: Kc inicial, Kc media y Kc final.

Huella hídrica gris: es el componente de color gris en la huella hídrica para el cultivo de Lirio japonés (WFproc,gris,m3/ton) el cual se calculó como la cantidad aplicada de productos químicos para el campo por hectárea (AR, kg/ha) multiplicado por la fracción $(\mathbb{\nabla})$ de lixiviación y escorrentía, y dividido por la concentración máxima aceptable (Cmax, kg/m3), menos la concentración natural para el contaminante considerado (Cnat, $\mathrm{kg} / \mathrm{m} 3$ ) $\mathrm{y}$ finalmente dividido por el rendimiento del cultivo (Y, ton/ha) (Ecuación 4). 
Para los cálculos de la huella hídrica gris fue necesaria la determinación de contaminantes que son producto de la lixiviación por la utilización de agroquímicos, tales como, fertilizantes, plaguicidas o pesticidas en el proceso productivo y el cual hace referencia a la fracción de lixiviación.

Otra de las variables incluidas en este componente es la concentración máxima aceptable que hace referencia a la concentración de agroquímicos permitidos por la legislación colombiana; para esto fue necesario tener en cuenta las concentraciones permitidas por la Resolución 1848 del 2014 y la Resolución 631 del 2015.

La huella hídrica total para el cultivo de Lirio japonés se determinó a partir de la suma de los componentes verde, azul y gris (Ecuación 5).

Finalmente, en la tercera fase se realizó el análisis de los resultados obtenidos los cuales se compararon con algunos de los estudios nacionales de huellas hídricas de diversos cultivos de flores, con el ánimo de indicar la magnitud del impacto que tiene el cultivo de Lirio japonés sobre el recurso hídrico.

\section{Resultados y Análisis}

En la Tabla II se muestran los resultados de los valores obtenidos de las huellas hídricas verde y azul, los cuales fueron hallados mediante información suministrada por la administración del cultivo. Para la huella hídrica gris, Tabla III, se tuvo en cuenta la cantidad de nitrato de potasio aplicado en el cultivo.

TABLA ॥

Huella Hídrica Azul y Verde Gris Para el Cultivo de LIRIO JAPONÉS

\begin{tabular}{l|c|c}
\hline $\begin{array}{l}\text { Lirio } \\
\text { Japo- } \\
\text { nés }\end{array}$ & $\begin{array}{c}\text { Huella Hídrica Azul } \\
\text { (L/tallo) }\end{array}$ & $\begin{array}{c}\text { Huella Hídrica } \\
\text { Verde (L/tallo) }\end{array}$ \\
\hline
\end{tabular}

TABLA III

Huella Hídrica Gris Para el Cultivo de Lirio Japonés

Lirio Japonés

Huella Hídrica Gris (L/tallo)

$2.06 \times 10-4$

La huella hídrica total del cultivo de Lirio japonés se muestra en la Tabla IV y en la Fig. 3, que corresponde a la suma de los tres componentes determinados anteriormente ( $\mathrm{HH}$ verde, $\mathrm{HH}$ azul, $\mathrm{HH}$ gris).

TABLA IV

Huella Hídricas Para el Cultivo de Lirio Japonés

\begin{tabular}{c|c|c|c|c|}
\hline & $\begin{array}{c}\text { Huella Hídrica Azul } \\
\text { (L/tallo) }\end{array}$ & $\begin{array}{c}\text { Huella Hídrica Ver- } \\
\text { de (L/tallo) }\end{array}$ & $\begin{array}{c}\text { Huella Hídrica Gris } \\
\text { (L/tallo) }\end{array}$ & $\begin{array}{c}\text { Huella Hídrica Total } \\
\text { (L/tallo) }\end{array}$ \\
\hline \multirow{2}{*}{ Lirio Japonés } & $1.76 \times 10-4$ & $1 \times 10-8$ & $2.06 \times 10-4$ & $3,82 \times 10-4$ \\
\hline
\end{tabular}

Para la huella hídrica verde se tuvo en cuenta el volumen de agua lluvia que es aprovechado en el cultivo. Para tal fin, se tomó un valor del 5\%, el cual es un valor arbitrario debido a que en el cultivo no se realiza el aprovechamiento de esta agua. Se tiene en cuenta que puede haber algún tipo de aprovechamiento de la misma. El valor hallado se encuentra expresado en la Tabla I.
En cuanto a la huella hídrica azul se sabe que esta representa el volumen de agua que el cultivo consume y la cual proviene de fuentes superficiales y/o subterráneas; esta se utiliza a lo largo de la cadena productiva y se evapora en este mismo proceso. En el caso específico de este cultivo, toda el agua necesaria para el riego del mismo se toma de la quebrada Puerquera. Es importante 
tener en cuenta que para la determinación de esta huella, se realiza el riego del cultivo cada dos días con un volumen de agua de 20 litros por cada uno de los surcos, contando con un total de 15 surcos, para un total de 70 litros por semana. En relación con el volumen de agua empleado para el riego del cultivo se estimó que el 95\% se utiliza para el proceso productivo; vale aclarar que se pueden tener pérdidas en el proceso de transporte del agua desde su captación hasta llegar al sistema de riego, contando con que el $5 \%$ de agua lluvia se utilice en el cultivo.

Al realizar una comparación de la huella hídrica del cultivo de Lirio japonés con otro cultivo, como por ejemplo el del sector arrocero, de gran impacto en la región, se concluye que el consumo de agua por parte del primero es relativamente mínimo al consultado en este sector arrocero, ya que este cuenta con unos valores de huellas hídricas verde y azul en el ámbito nacional del $12 \%$ y el $41 \%$, respectivamente.

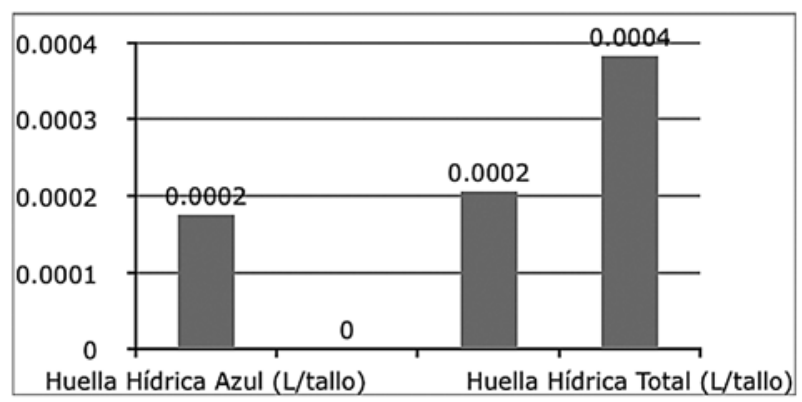

Fig. 3 Huellas hídricas del cultivo de Lirio Japonés.

El valor de la huella hídrica gris es de 2.0625×10-4 L/tallo; esto puede deberse a que en el proceso de cultivo se aplica 2 veces $5 \mathrm{~g} / \mathrm{L}$ de nitrato de potasio. Para una mejor aproximación del análisis de la huella se tienen en cuenta los valores de los parámetros medidos en campo (Tabla V), en comparación con la Resolución 1848 del 2014, en los cuales puede verse que el valor del nitrato cumple con el valor permisible tanto en la salida como en la entrada. Debe tenerse en cuenta que la muestra fue tomada de la quebrada La Puerquera, para cultivos agrícolas restringibles, que se encuentra entre 50 y 100 $\mathrm{mg} / \mathrm{L}$. El valor de nitritos no cumple con el valor que nos da la resolución $(0.1 \mathrm{mg} / \mathrm{L}$, también tomado para cultivos agrícolas restringibles). Esto puede deberse a las condiciones naturales del cuerpo de agua, porque si se comparan los valores de nitritos con nitratos puede verse que los nitritos son más altos tanto en la entrada como en la salida, sabiendo que estos cumplen el ciclo del nitrógeno y que los nitratos pasan a nitritos. Es por ello que existe esa diferencia. Para el vaIor de la DQO se tuvo en cuenta la Resolución 631 del 2015, capítulo VII, en la cual dice que el valor permisible es de $150 \mathrm{mg} / \mathrm{LO} 2$. Puede verse que se cumple con el valor de la norma tanto en la salida como en la entrada.

TABLA V

Parámetros Medidos En Campo

\begin{tabular}{l|c|c|}
\multicolumn{1}{|c|}{ Parámetro } & Entrada (mg/L) & Salida (mg/L) \\
\hline Nitritos & 13 & 4 \\
\hline Nitratos & 1.9 & 2.7 \\
\hline DQO & 37 & 76 \\
\hline
\end{tabular}

El resultado de la huella hídrica total es la suma de las tres huella anteriores (azul, verde y gris), valor que hace referencia a la cantidad de agua utilizada para el proceso de cultivo de Lirio japonés, el cual es 3,82×10-4L/Tallo(Tabla III). El valor de esta huella hídrica total puede compararse con la huella hídrica por producción de flores que se encuentra en el documento Una mirada a la agricultura de Colombia desde su huella hídrica [3], y que es de aproximadamente $900 \mathrm{~m} 3 / \mathrm{Ton}$, mientras que la del Lirio japonés es de $7.64 \times 10-3 \mathrm{~m} 3 /$ ton, aportando aproximadamente el $0.00085 \%$ del total de la huella hídrica por producción de flores. 


\section{V.Conclusiones}

Mediante el diagnóstico realizado al proceso productivo del cultivo de Lirio japonés, se puede evidenciar que la finca cuenta con sistemas de aprovechamiento de residuos sólidos orgánicos (como el compostaje) generados en los procesos productivos de las diferentes plantaciones que se encuentran en los predios de la misma, lo cual permite que se minimice el impacto de la aplicación de agroquímicos para el mejoramiento de las condiciones naturales del suelo.

El modelamiento en el software Cropwat 8.0 fue fundamental en el cálculo de las diferentes huellas hídricas, por lo que puede concluirse que es un programa útil para determinar la evapotranspiración de los cultivos, los requerimientos de agua de los cultivos que pueden utilizar los ingenieros ambientales y otros profesionales que estén interesados en el tema.

Se realizó el cálculo de las huellas hídricas verde, azul y gris para el cultivo de Lirio japonés, cuyos valores son $1 \times 10-8 \mathrm{~L} /$ Tallo, $1.76 \times 10-4 \mathrm{~L} / \mathrm{Ta}-$ llo y $2.06 \times 10-4 \mathrm{~L} /$ Tallo, respectivamente. Estos datos, al compararlos con las huellas hídricas de diferentes procesos productivos del país, resultan ser mínimos debido a que el volumen de agua utilizado en el proceso no es significativo, pues solo representa el $0.00085 \%$ del total de la huella hídrica nacional [13].

La huella hídrica total del cultivo de Lirio japonés (7.64x10-3m3/ton) en comparación con la huella hídrica por producción de flores $(900 \mathrm{~m} 3 /$ Ton), aporta tan solo el $0.00085 \%$ del total, por lo cual puede concluirse que este cultivo es uno de los que tiene un menor aporte en dicha huella total para la producción de flores en Colombia [14].

\section{REFERENCIAS}

[1] Corpoguavio, "Esquema de Ordenamiento Territorial Boyacá"-Boyacá, Colombia, 2011.

[2] Norma Internacional ISO 14046:2014.

[3] D. Arevalo, "A look atcolombian agriculture from its water footprint", World Wildlife Fund WWF. Cali, Colombia, 2012.

[4] WWAP, "The United Nations World Water Development Report” 3. UN World Water Assessment Programme, 2009.

[5] A. Chapagain and A. Hoekstra, "Globalization of Water: Sharing the Planet's Freshwater Resources", 2008.

[6] A. Hoekstra, "Human appropriation of natural capital: A comparison of ecological footprint and water footprint analysis", 2009.

[7] A. Hoekstra, A. Chapagain, M. Aldaya and M. Mekonne, "The water footprint assessment manual: setting the global standard, Earthscan", Washington, DC, 2011.

[8] A. Hoekstra, "Virtual water trade, Value of Water Research Report Series No.12", Febrero de 2003.

[9] F. Bermúdez, "El fin del fin, Centro de estudios Políticos e Investigaciones Históricas", Editorial Panamericana, Bogotá Colombia, 2007.

[10] Ecopetrol, "Instructivo para el muestro de suelos sedimentos y lodos", 2011.

[11] R. Allen, L. Pereira, D. Raes, and M. Smith, "Evapotranspiración del cultivo, Guías para la determinación de los requerimientos de agua de los cultivos.' Organización de las naciones unidas para la agricultura y la alimentación. Roma. Italia, 2006.

[12] FAO, O. d.. Manual Cropwat 8.0, 2010.

[13] Y. Vanegas and L. Ramírez, "Evaluación de la huella hídrica para la producción de flores lirio japonés $(\mathrm{He}-$ merocallis) en la vereda Ricaurte del municipio de Rondón - Boyacá" Trabajo de grado Ingeniera Ambiental y Sanitaria, Universidad de La Salle, Bogotá, Colombia, 2015.

[14] W. Baez, ,"Evaluación de la huella hídrica en la producción de clavel estándar (Dianthus caryophyllus) en la sabana de Bogotá," Tesis Gerencia Ambiental, Universidad Libre, Bogotá, Colombia, 2013. 
